\title{
Heavy ions at LHC energies: Predictions for net- baryon distributions
}

\author{
Yacine Mehtar-Tani ${ }^{1}$, Georg Wolschin ${ }^{2}$ * \\ ${ }^{1}$ Departamento de Física de Partículas and IGFAE, Universidade de Santiago de Compostela, \\ Spain \\ ${ }^{2}$ Institut für Theoretische Physik der Universität, Philosophenweg 16, D-69120 Heidelberg, \\ Germany
}

DOI: http://dx.doi.org/10.5689/UA-PROC-2010-09/51

\begin{abstract}
Baryon transport in relativistic heavy-ion collisions is investigated within a gluon saturation model. Predictions are made for net-baryon and net-proton rapidity distributions in central $\mathrm{Pb}+\mathrm{Pb}$ collisions at CERN LHC energies of up to $\sqrt{s_{N N}}=5.5 \mathrm{TeV}$ both at forward rapidities, and in the central rapidity region where data will soon be available.
\end{abstract}

\section{Introduction}

With the CERN Large Hadron Collider LHC now in operation for proton-proton collisions at center-of-mass energies of up to $7 \mathrm{TeV}[1,2]$, the advent of heavy-ion collisions at the LHC is expected in November 2010, and corresponding theoretical predictions will then be tested. In this note such predictions for selected observables will be presented.

We calculate rapidity distributions of net baryons, and net protons $(p-\bar{p})$ in central heavyion collisions using an approach that is based on quantum chromodynamics and in particular, on the gluon saturation model $[3,4,5,6]$. Our specific model for net baryons is described in detail in $[7,8,9]$. A complementary phenomenological nonequilibrium-statistical model that is not based on QCD had been been formulated earlier [10] to predict the corresponding observables at LHC energies — and also charged-hadron pseudorapidity distributions — with similar results for net baryons.

The calculations for net-baryon rapidity distributions within our QCD-based model are reviewed in Sec. 2, and results at LHC energies are given in Sec. 3. Conclusions are drawn in Sec. 4.

\section{Rapidity distributions for net baryons based on QCD}

Gluon saturation has been the focal point of important and interesting particle-physics investigations since many years. Its observation would provide access to a new regime of quantum chromodynamics where high-density gluons form a coherent state. In regions of large parton

*Speaker; E-mail: wolschin@uni-hd.de 
densities the physics is governed by a single hard scale $Q_{s} \gg \Lambda_{\mathrm{QCD}}$ which increases with energy and thus allows the use of small-coupling techniques $[3,4,5,6]$. In this regime gluon recombination starts to compete with the exponentially increasing gluon splitting, and the gluon distribution function is expected to saturate.

At the HERA, some evidence for gluon saturation in the proton had been found in deep inelastic $e+p$ collisions at high energy and low values of Bjorken- $x$, but the results are still open to interpretation [11]. The existence of geometric scaling as predicted by the color glass theory as an approach to saturation physics had indeed been confirmed, constituting the most important evidence for saturation so far [12].

Since the saturation scale is enhanced by a factor $A^{1 / 3}$ in heavy ions as compared to protons, it is natural to investigate gluon saturation in relativistic heavy-ion collisions, as has been done by many authors [13]. Most theoretical investigations concentrate on charged-hadron production from inclusive gluon interactions, and in the central rapidity region a reasonable understanding has been achieved in the color glass condensate framework $[6,14,15,16]$ through inclusive gluon production $[17,18]$. However, the valence-quark scattering off the gluon condensate as an observable in net-baryon distributions $[7,8]$ is expected to provide interesting new information on gluon saturation, and on geometric scaling [12].

Here the most promising effects arise at very forward angles, or correspondingly large values of the rapidity $y \simeq 5-8$ at $\mathrm{LHC}$ energies of $\sqrt{s_{N N}}=5.5 \mathrm{TeV}$ for $\mathrm{Pb}+\mathrm{Pb}$, with a beam rapidity of 8.68. For symmetric systems, two symmetric fragmentation peaks are expected to be present in the net-baryon distributions at forward/backward rapidities, as was shown in the RDM-calculations [10]. In particular, we have discussed in [7] that it is in principle possible to determine the growth of the saturation-scale, $\lambda \equiv d \ln Q_{s} / d y_{b}$ from the position of the fragmentation peak in rapidity space. Here the beam rapidity is $y_{\mathrm{b}}=1 / 2 \cdot \ln \left[\left(E+p_{L}\right) /(E-\right.$ $\left.\left.p_{L}\right)\right] \simeq \ln (\sqrt{s} / m)$ at beam energy $E$ and longitudinal momentum $p_{L}$ with the nucleon mass $m$.

In the region of large values of Bjorken- $x$ the valence-quark parton distribution in the projectile is well-known, and can hence be used to access the gluon distribution at small $x$ in the other nucleus where saturation is expected to occur due to the competition of gluon recombination with the exponentially increasing gluon splitting [14, 15, 19, 20, 16].

For the next years of LHC operation, however, experimental investigations of central heavyion collisions with particle identification will concentrate on the midrapidity region $y \leq 2$ and hence, we also focus our predictions for net-baryon distributions at LHC energies to this region. It turns out that here the saturation-scale exponent $\lambda$ can not be determined from net-baryon distributions because the dependence of the distribution function on $\lambda$ is too weak near midrapidity.

The differential cross section for valence quark production in a high-energy nucleus-nucleus collision is calculated from $[21,22,23]$

$$
\frac{d N}{d^{2} p_{T} d y}=\frac{1}{(2 \pi)^{2}} \frac{1}{p_{T}^{2}} x_{1} q_{v}\left(x_{1}, Q_{f}\right) \varphi\left(x_{2}, p_{T}\right),
$$

where $p_{T}$ is the transverse momentum of the produced quark, and $y$ its rapidity. The longitudinal momentum fractions carried, respectively, by the valence quark in the projectile and the soft gluon in the target are $x_{1}=p_{T} / \sqrt{s} \exp (y)$ and $x_{2}=p_{T} / \sqrt{s} \exp (-y)$. The factorization scale is usually set equal to the transverse momentum, $Q_{f} \equiv p_{T}$. We have discussed the gluon distribution $\varphi\left(x, p_{T}\right)$ and details of the overall model in [7] .

One important prediction of the color glass condensate theory is geometric scaling: the gluon distribution depends on $x$ and $p_{T}$ only through the scaling variable $p_{T}^{2} / Q_{s}^{2}(x)$, where 
$Q_{s}^{2}(x)=A^{1 / 3} Q_{0}^{2} x^{-\lambda}, A$ is the mass number and $Q_{0}$ sets the dimension. This has been confirmed experimentally at HERA [12] . The fit value $\lambda=0.2-0.3$ agrees with theoretical estimates based on next-to-leading order Balitskii-Fadin-Kuraev-Lipatov (BFKL) results [24, 25]. To show that the net-baryon distribution reflects the geometric scaling of the gluon distribution, the following change of variables has been proposed in [7]: $x \equiv x_{1}, x_{2} \equiv x e^{-2 y}, p_{T}^{2} \equiv x^{2} s e^{-2 y}$. Thus after integrating over $p_{T}$, the rapidity distribution can be rewritten as

$$
\frac{d N}{d y}(\tau)=\frac{C}{2 \pi} \int_{0}^{1} \frac{d x}{x} x q_{v}(x) \varphi\left(x^{2+\lambda} e^{\tau}\right),
$$

where $\tau=\ln \left(s / Q_{0}^{2}\right)-\ln A^{1 / 3}-2(1+\lambda) y$ is the corresponding scaling variable. Hence, the net-baryon multiplicity in the peak region is only a function of a single scaling variable $\tau$, which relates the energy dependence to the rapidity and mass number dependence. In the fragmentation region, the valence quark distribution is only very weakly dependent on $Q_{f}$.

\section{Predictions at $\mathrm{LHC}$ energies}

A prediction for central $\mathrm{Pb}+\mathrm{Pb}$ at the maximum LHC energy of $5.52 \mathrm{TeV}$ is shown in Fig. 1 for $\lambda=0,0.15$, and 0.3 . At LHC energies the mid-rapidity region is almost baryon free, we obtain $d N / d y(y=0) \simeq 4$ for net baryons at $5.52 \mathrm{TeV}$. The position of the fragmentation peak is very sensitive to the value of $\lambda$, with a difference of about 1.5 units of rapidity between the $\lambda=0$ and 0.3 cases. It is possible that the full scaling regime with $\lambda$ approaching 0.3 can be reached at or beyond LHC energies, but presently none of the LHC-experiments is capable of measuring identified protons or neutrons from central $\mathrm{Pb}+\mathrm{Pb}$ collisions in the region of the fragmentation peaks. This would be a relevant proposal for future extensions of the detector capabilities at LHC.

With increasing energy (such as from RHIC to LHC) the peaks move apart, the solutions behave like travelling waves in rapidity space, which can be probed experimentally at distinct values of the beam energy, or the corresponding beam rapidity. the peak position as a function of the beam rapidity is $y_{\text {peak }}=v y_{b}+$ const with the peak velocity $v=1 /(1+\lambda)$. The position of the peak in rapidity space as a function of the beam energy can in principle be determined experimentally, or at least estimated (RHIC). Theoretically, its evolution with energy provides a measure of the saturation scale exponent $\lambda$. Hence, a precise determination of the net-baryon fragmentation peak position as a function of beam energy would provide detailed information about the gluon saturation scale.

The mean rapidity loss $\langle\delta y\rangle=y_{b}-\langle y\rangle$ is also shown in Fig. 1(b), our result is in agreement with the experimental values of baryon stopping that have been obtained at AGS, SPS and RHIC energies [26, 27, 28, 29]. Assuming that the mean rapidity evolves similarly to the peak position, $\langle y\rangle \equiv y_{\text {peak }}+$ const, the mean rapidity loss increases linearly at large $y_{b},\langle\delta y\rangle=$ $\frac{\lambda}{1+\lambda} y_{b}+$ const: the slope is related to $\lambda$.

Hence, the mean rapidity loss that accompanies the energy loss in the course of the slowdown of baryons also provides a potential measure for $\lambda$ and thus, a test of saturation physics. The grey band in Fig. 1(b) reflects the uncertainty of how to place the remaining baryons that are missing in the present model.

Our result for the mean rapidity loss as function of beam rapidity, or center-of-mass energy, emphasizes the importance of a detailed measurement at LHC energies to allow more definite 
conclusions about the value of $\lambda$ from net-baryon distributions in relativistic heavy-ion physics.
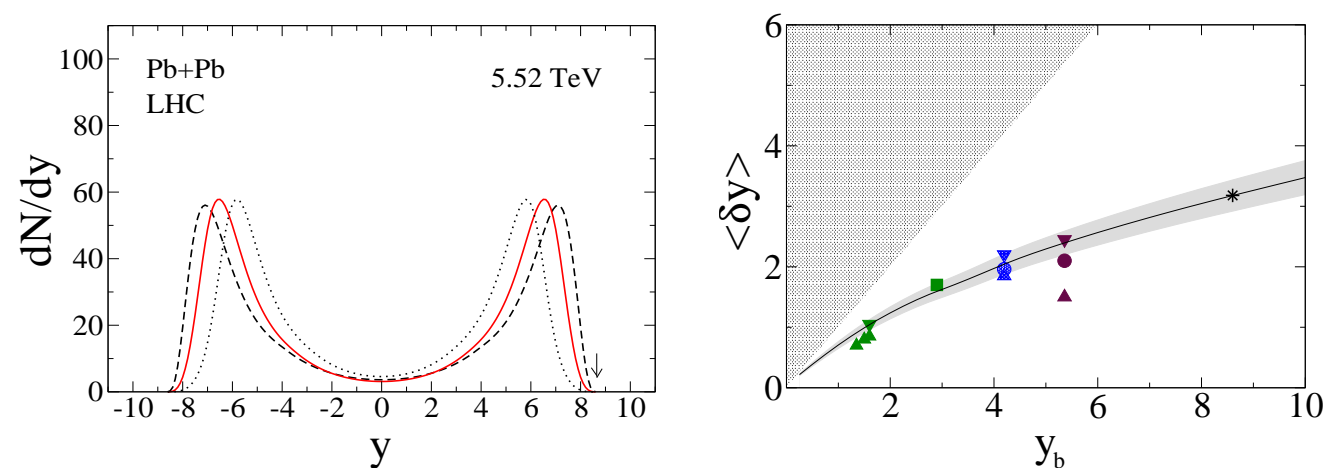

Figure 1: Central collisions of $\mathrm{Pb}+\mathrm{Pb}$ and $\mathrm{Au}+\mathrm{Au}$. (a) Rapidity distribution of net baryons in central $\mathrm{Pb}+\mathrm{Pb}$ collisions at LHC energies of $\sqrt{s_{N N}}=5.52 \mathrm{TeV}$. Theoretical distributions are shown over the full rapidity range including the fragmentation peaks for $\lambda=0$ (dashed), $\lambda=0.15$ (solid), and $\lambda=0.3$ (dotted curve). (b): The mean rapidity loss $\langle\delta y\rangle$ as obtained from our theoretical results is plotted as a function of beam rapidity $y_{b}$, solid curve. The star at $y_{\text {beam }}=8.68$ is our prediction for central $\mathrm{Pb}+\mathrm{Pb}$ at LHC-energies of $\sqrt{s_{N N}}=5.52 \mathrm{TeV}$ with $\lambda=0.2$ and $Q_{0}^{2}=0.04 \mathrm{GeV}^{2}$. Analysis results from AGS Au + Au data (E917, E802/E866, triangles) [26], SPS Pb + Pb data (NA49, square) [27], RHIC Au + Au data (BRAHMS, dots, with triangles as lower and upper limits) $[28,30]$ are compared with the calculations. From Mehtar-Tani and Wolschin $[7,8]$.

Since net-baryon data in central heavy-ion collisions at large rapidities will not be available for the next years, one first has to concentrate on the midrapidity region. We have made predictions in [9]. The LHC physics program starts with a center-of-mass proton-proton energy of $7 \mathrm{TeV}$, the corresponding energy for $\mathrm{Pb}+\mathrm{Pb}$ (scaling with $\mathrm{Z} / \mathrm{A}$ ) is $\sqrt{s_{N N}}=2.76 \mathrm{TeV}$. Results for the highest attainable $\mathrm{Pb}+\mathrm{Pb}$ energy of $5.52 \mathrm{TeV}$ have also been discussed in [9].

We have used the analytical form for $d N / d y=a \cosh (b y)$ in a direct comparison with BRAHMS net-proton data in central $\mathrm{Au}+\mathrm{Au}$ collisions at RHIC energies of $\sqrt{s_{N N}}=0.2$ $\mathrm{TeV}[28]$ through a $\chi^{2}-$ minimization of the two parameters $a$ and $b$, where $b=2 \Delta(1+$ $\lambda) /(2+\lambda)$, and $\Delta \simeq 0.47$ is related to a strong-coupling constant $\alpha_{s} \simeq 0.3[9]$.

Our comparison with the BRAHMS $\mathrm{Au}+\mathrm{Au}$ data in the midrapidity region is shown in Fig. 2 for net protons. We display the energy dependence of our net-proton central $\mathrm{Pb}+\mathrm{Pb}$ predictions at LHC energies for $\sqrt{s_{N N}}=2.76,3.94$, and $5.52 \mathrm{TeV}$. At $y=0$ the corresponding values of $d N / d y$ are $1.9,1.7$, and 1.4 .

A description for the net-proton rapidity distribution within the relativistic diffusion model (which is not based on QCD, but on nonequilibrium-statistical physics) had been developed in [10]. There the predicted midrapidity value for central $\mathrm{Pb}+\mathrm{Pb}$ at the LHC energy of $\sqrt{s_{N N}}$ $=5.52 \mathrm{TeV}$ is $d N / d y \simeq 1-2.5$ depending on the model parameters and hence, comparable to the present QCD-based result. 


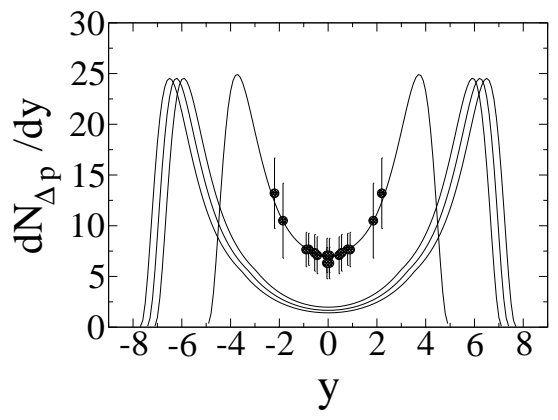

Figure 2: Calculated rapidity distributions of net protons in $0 \%-5 \%$ central $\mathrm{Pb}+\mathrm{Pb}$ collisions at LHC energies of $\sqrt{s_{N N}}=2.76,3.94,5.52 \mathrm{TeV}$. Our result for central $\mathrm{Au}+\mathrm{Au}$ collisions at RHIC energies of $0.2 \mathrm{TeV}$ is compared with BRAHMS data [28] in a $\chi^{2}-$ minimization. From Mehtar-Tani and Wolschin [9].

\section{Conclusion}

To conclude, predictions for net-baryon (proton) distributions in central $\mathrm{Pb}+\mathrm{Pb}$ collisions at LHC energies have been presented. The investigation uses a microscopic approach based on quantum chromodynamics. This transparent QCD-based model [7,8] with well-established parton distribution functions in the context of saturation physics allows to calculate transverse momentum [8] and rapidity distributions for net baryons, and also net kaons [7, 8, 9].

The underlying physical process is the scattering of valence quarks off the gluon condensate in the respective other nucleus. In the forward rapidity region the position of the fragmentation peak is very sensitive to the gluon saturation scale, such that the saturation-scale exponent could in principle be determined from LHC data should these become accessible in the coming years. In the near future, net-baryon (proton) data in central $\mathrm{Pb}+\mathrm{Pb}$ collisions at LHC energies will, however, only be available near midrapidity $|y|<2$. In this region detailed results have been presented, which will soon be compared to LHC heavy-ion data.

\section{Acknowledgments}

This work is supported by the ExtreMe Matter Institute, EMMI.

\section{References}

[1] K. Aamodt et al. (ALICE Collaboration), Eur. Phys. J. C 65, 111 (2010).

[2] V. Khachatryan et al. (CMS Collaboration), JHEP 02, 041 (2010).

[3] L. V. Gribov, E. M. Levin, and M. G. Ryskin, Phys. Rep. 100, 1 (1983).

[4] A. H. Mueller and J. Qiu, Nucl. Phys. B268, 427 (1986).

[5] J. P. Blaizot and A. H. Mueller, Nucl. Phys. B289, 847 (1987). 
[6] L. McLerran and R. Venugopalan, Phys. Rev. D 49, 2233 (1994).

[7] Y. Mehtar-Tani and G. Wolschin, Phys. Rev. Lett. 102, 182301 (2009).

[8] Y. Mehtar-Tani and G. Wolschin, Phys. Rev. C 80, 054905 (2009).

[9] Y. Mehtar-Tani and G. Wolschin, Phys. Lett. B 688, 174 (2010).

[10] G. Wolschin, Prog. Part. Nucl. Phys. 59, 374 (2007).

[11] V. Šimák et al. (eds.), AIP Conf. Proc. 828, 339 (2006).

[12] A. M. Staśto, K. Golec-Biernat, and J. Kwieciński, Phys. Rev. Lett. 86, 596 (2001).

[13] N. Armesto et al. (eds.), J. Phys. G 35, 054001 (2008).

[14] I. Balitsky, Nucl. Phys. B463, 99 (1996).

[15] J. Jalilian-Marian, A. Kovner, A. Leonidov, and H. Weigert, Nucl. Phys. B504, 415 (1997).

[16] E. Iancu, A. Leonidov, and L. D. McLerran, Nucl. Phys. A692, 583 (2001).

[17] D. Kharzeev, E. Levin, and M. Nardi, Nucl. Phys. A747, 609 (2005).

[18] J. L. Albacete, Phys. Rev. Lett. 99, 262301 (2007).

[19] J. Jalilian-Marian, A. Kovner, and H. Weigert, Phys. Rev. D 59, 014015 (1998).

[20] J. Jalilian-Marian, A. Kovner, A. Leonidov, and H. Weigert, Phys. Rev. D 59, 034007 (1999).

[21] D. Kharzeev, Y. V. Kovchegov, and K. Tuchin, Phys. Lett. B599, 23 (2004).

[22] R. Baier, Y. Mehtar-Tani, and D. Schiff, Nucl. Phys. A764, 515 (2006).

[23] A. Dumitru, A. Hayashigaki, and J. Jalilian-Marian, Nucl. Phys. A765, 464 (2006).

[24] L. N. Lipatov, Sov. J. Nucl. Phys. 23, 338 (1976).

[25] D. N. Triantafyllopoulos, Nucl. Phys. B648, 293 (2003).

[26] F. Videbaek and O. Hansen, Phys. Rev. C 52, 2684 (1995).

[27] H. Appelshäuser et al. (NA49 Collaboration), Phys. Rev. Lett. 82, 2471 (1999).

[28] I. G. Bearden et al. (BRAHMS Collaboration), Phys. Rev. Lett. 93, 102301 (2004).

[29] I. C. Arsene et al. (BRAHMS Collaboration), Phys. Lett. B677, 267 (2009).

[30] H. H. Dalsgaard et al. (BRAHMS Collaboration), Int. J. Mod. Phys. E 16, 1813 (2007). 